\title{
The Neutrophil Response to Rabbit Antimicrobial Extract After Implantation of Biomaterial into a Bone/Cartilage Defect
}

\author{
TOMASZ SZPONDER ${ }^{1}$, JOANNA WESSELY-SZPONDER ${ }^{2}$ and ALEKSANDRA SOBCZYŃSKA-RAK ${ }^{1}$ \\ ${ }^{1}$ Department and Clinic of Animal Surgery, and ${ }^{2}$ Department of Pathophysiology, \\ Faculty of Veterinary Medicine, University of Life Sciences, Lublin, Poland
}

\begin{abstract}
Background/aim: In this study, the neutrophil response to an antimicrobial extract was evaluated as a potential marker of inflammatory process after implantation of alginate and carbon fiber biomaterials into a bone or cartilage defect in rabbits. Materials and Methods: The response to biomaterials used was assessed based on enzyme release, generation of reactive oxygen species (ROS) and survival of neutrophils isolated from the rabbit's blood after implantation. Results: The implantation of alginate biomaterial increased elastase and alkaline phosphatase release, whereas carbon fibers did not evoke increased elastase release. The implantation of both biomaterials resulted in a higher myeloperoxidase (MPO) release after 30-min incubation. Stimulation with different fractions of antimicrobial peptide (AMP) extract diminished MPO release and nitric oxide generation, as well as slightly reducing neutrophil survival. Conclusion: Our study permits the assessment of the neutrophil intravital response to an implant without the need for preparation of histological sections. Additionally, AMP extract restricted some manifestations of the pro-inflammatory response and may be considered a regulator of neutrophil activity in the early inflammatory phase, preventing rejection of the implant.
\end{abstract}

Biomaterials are implanted into the body tissue for a variety of therapeutic applications, and the immune system initiates a series of actions including the activation of inflammatory cells, which ultimately influence the effect of the implantation (1). Numerous innate immune cells participate in a foreign

This article is freely accessible online.

Correspondence to: Joanna Wessely-Szponder, Department of Pathophysiology, Chair of Preclinical Veterinary Sciences, Faculty of Veterinary Medicine, Akademicka 12, 20-033 Lublin, Poland. Tel: +48 814456774, Fax: +48 814456024, e-mail: joanna.wessely@up.lublin.pl

Key Words: Biomaterial, alginate, carbon fibers, antimicrobial peptides, neutrophils. body response against these implants. While the potential role of mast cells, monocytes, and macrophages in this process has already been described, the precise role of neutrophils remains unclear (2). Neutrophils are the leading cells in the first line of cellular response to many different stimulations. Their role is particularly important in the first inflammatory phase of soft-tissue and bone repair; they exert chemotactic effects involved in the recruitment of further inflammatory and mesenchymal cells, in stimulating angiogenesis, and in enhancing extracellular matrix synthesis (3). A recent study revealed that apart from the above-mentioned functions, these cells are involved in other immune responses at sites of tissue injury. For example, neutrophils undergo a process of cell death that leads to the release of intranuclear DNA into the extracellular space, forming neutrophil extracellular traps (2). Activated neutrophils degranulate and release proteolytic enzymes such as elastase and various reactive oxygen species (ROS), these compounds are important in host defense against invading organisms, however, they can also lead to secondary tissue and organ damage (4).

Contrary to this, it has been suggested that neutrophils have some suppressive functions, which might help with the resolution of inflammation, and potentially promote tissue growth (5). These new findings have raised questions regarding the exact nature of neutrophil-biomaterial interactions, creating the possibility to use suppressive functions of neutrophils to reduce inflammatory reactions to biomaterials (5). In order to assess this effect, our study examined the response of neutrophils isolated from blood after the implantation of an alginate implant into a bone, or carbon fiber into a cartilage defect, in rabbits, as two different biomaterials implanted into different tissues.

The new promising therapeutic approach for regulation of the intensity of the inflammatory process are antimicrobial peptides (AMPs), cathelicidins and defensins, with their potential for immunomodulation (6-8). Previous reports estimated that some cathelicidins act as immunomodulators by increasing or diminishing the immune response, including having an influence on neutrophils (9). They can change neutrophil degranulation and can regulate inflammatory 
response by promoting or decreasing reducing apoptosis as the means of modulating inflammation (10). Defensins, in turn, apart from their bactericidal, antifungal and antiviral activity, exert immunomodulatory activity e.g. by the modification of cell death (9).

For this experiment, a crude neutrophil extract and its two fractions [high molecular mass fraction 1 (F1) and low molecular mass fraction 2 (F2)] were used. As determined in our previous work (11), F1 of the neutrophil extract consisted of rabbit cathelicidins of higher molecular masses, namely $15 \mathrm{kDa}$ AMP (a mass of 15,626 Da), cathelin-like fragments of about $11,000 \mathrm{Da}$, and products of fragmentation of rabbit cathelicidin CAP 18. In contrast, in F2 of low molecular masses, defensins from rabbit neutrophils, namely NP-1, NP-2, NP-3a, NP-3b, NP-4 and NP-5, were detected. Crude neutrophil extract contained all these components mixed together.

The aim of this study was to evaluate the influence of different fractions of rabbit neutrophil AMP extract as potential inflammatory regulators on rabbit neutrophils isolated during implantation of two kinds of biomaterials: alginate-based biomaterial into a bone, and carbon fiber into a cartilage defect.

\section{Materials and Methods}

Preparation of AMP. AMP was isolated according to the method described previously (12). In brief, fresh porcine blood was collected with $3.8 \%$ citrate as anticoagulant at an abattoir. After red blood cell lysis with $0.83 \%$ ammonium chloride, the blood was centrifuged at $700 \times g$ for $15 \mathrm{~min}$ at $4^{\circ} \mathrm{C}$. The neutrophils obtained (75-80\% pure) were resuspended in a modified phosphate saline buffer, and then homogenized with a DIAX 900 Heidolph (SigmaAldrich, Poznań, Poland) (12.5 rpm, $15 \mathrm{~min})$ to release the neutrophil granules. These granules were collected $(25,000 \times g$ for $40 \mathrm{~min}$ at $4^{\circ} \mathrm{C}$ ), suspended in $10 \%$ acetic acid and stirred overnight at $4^{\circ} \mathrm{C}$ to extract AMPs. The solution containing the peptides was separated from the granules $\left(25,000 \times g\right.$ for $20 \mathrm{~min}$ at $\left.4^{\circ} \mathrm{C}\right)$, lyophilized and stored at $-20^{\circ} \mathrm{C}$.

Gel filtration chromatography was used to separate the components present in the crude extract according to their molecular mass. The extract was passed through a Sephadex G-50 column (Fine; Sigma-Aldrich) using a running buffer of $5 \%$ acetic acid at $0.5 \mathrm{ml} / \mathrm{min}$. The absorbance of the eluate (every $1 \mathrm{ml}$ ) was monitored at $280 \mathrm{~nm}$. The concentration of peptides in F1 and in F2 were assessed using the extinction coefficient.

Methods for alginate and carbon fiber characterization. Biomaterials implanted in the tibia of experimental animals (New Zealand rabbits) were fabricated from nanocomposite biopolymer fibers. The fibers were manufactured according to a procedure developed at the Department of Man-Made Fibers of the Lodz University of Technology, Poland. As a precursor, an aqueous spinning solution of sodium alginate (Protanal LF 10/60LS; FMC BioPolymer, Olsztyn, Poland) was used. Hydroxyapatite (3 wt.\%) with the molecular formula $\mathrm{Ca}_{10}\left(\mathrm{PO}_{4}\right)_{6}(\mathrm{OH})_{2}$, manufactured according to a procedure developed at the University of Science and
Technology (AGH-UST) Krakow, Poland, was introduced into the precursor solution and the whole was homogenized by sonication. The fibers were formed by a wet-spinning method where $\mathrm{CaCl}_{2}$ solution and ethyl alcohol were used consecutively as solidifying baths. In this way, hydroxyapatite-modified calcium alginate fibers were fabricated (13).

The carbon fibers used in the study were obtained from raw organic polymer precursor polyacrylonitrile (PAN) (Zoltek, Nyergsuijfalu Hungary). The polymer fibers were thermally converted in a two-stage process, the first stage was conducted in an atmosphere of air, the second in a protective nitrogen atmosphere.

Surgical procedures. Animals: Studies were conducted on 14 New Zealand White male rabbits, aged between 6 and 8 months, with mean \pm SD body weight of $4,000 \pm 500 \mathrm{~g}$. The animals were divided into two groups: an experimental and a sham group of 10 and 4 animals, respectively. In the sham group, drilled osteochondral $(n=2)$ or bone defect lesions $(n=2)$ were left without implantation and allowed to heal spontaneously. The experimental group comprised rabbits that underwent biomaterial implantation, and was divided into two subgroups of five rabbits for the implantation of alginate into tibial defects $(n=5)$ and five rabbits for the implantation of carbon fibers into knee joints $(n=5)$. The conditions of the experiment were approved by Local Ethics Committee No. II in Lublin (permit no. 19/2011 to conduct experiments on animals). Before surgery, all rabbits were put under general anesthesia using a mixture of ketamine $(30 \mathrm{mg} / \mathrm{kg}$, Vetaketam; Vet-Agro, Lublin, Poland) and xylazine (5 mg/kg, Sedazin; Biowet Puławy, Poland) injected intramuscularly. After approximately $15 \mathrm{~min}$, an intravenous cannula $(0.8 \times 25 \mathrm{~mm})$ was introduced into the marginal auricular vein. Aesthesia was maintained intravenously using the mixture mentioned above administered adequately.

Implantation of alginate-based biomaterial: The procedures were performed under standard sterile conditions. After hair removal, shaving, disinfection and draping, a straight 3-cm skin incision was made over the medial proximal tibia. After surgical exposure, a 4 $\mathrm{mm}$ defect was then made using an electric surgical drill. During drilling, the drill was continuously cooled with saline. Immediately before insertion of the implants, the hole was irrigated with saline to remove any bone fragments. Alginate biomaterial was then placed into the perforation and pressed into the surgical cavity until it was fixed to the cortical bone. The muscle tissue and skin were then sutured.

Implantation of carbon fiber biomaterial: A lateral approach to the knee joint was performed after preparation of the sterile field. Arthrotomy and medial patella dislocation allowed access to the femoral trochlea. A bone bur was used to drill a cylindrical hole into the patellar sulcus, $4 \mathrm{~mm}$ in diameter and approximately $5 \mathrm{~mm}$ in depth, reaching the subchondral bone. Next, the created defect was filled with carbon fiber implant by manually press-fitting it into place. After reducing the patella dislocation, a standard anastomosis procedure was performed on the articular capsule and the fascia lata, and then the subcutaneous tissue and skin.

After the surgeries, all of the rabbits were inspected daily for clinical signs of complications or adverse reactions. Three months after implantation, both implanted materials proved to be fully biocompatible, based on clinical, radiological, histopathological and scanning electron microscopic analyses (data not included). No adverse reactions were noted during the whole period of observation. 
Neutrophil cell culture. Blood samples from the rabbits were taken into tubes with EDTA at the following points in time: $48 \mathrm{~h}$ before and about $2 \mathrm{~h}$ after the surgical procedure. Neutrophils were isolated from the peripheral blood according to the procedure described for the preparation of the AMP extract. The number and viability of the obtained cells were determined using an R1 Automated Cell Counter (Olympus, Warsaw, Poland) and then the cells were plated at a density of $2.0 \times 10^{6}$ cells $/ \mathrm{ml}$.

The cell suspensions were supplemented as follows: the control group with phosphate-buffered saline (PBS; Biomed, Lublin, Poland), the crude neutrophil AMP group with AMP at a concentration of $20 \mu \mathrm{g} / \mathrm{ml}$, the F1 group with F1 at $40 \mu \mathrm{g} / \mathrm{ml}$, and the F2 group with F2 at $40 \mu \mathrm{g} / \mathrm{ml}$. The cultures were incubated for $30 \mathrm{~min}$ at $37^{\circ} \mathrm{C}$ and $5 \% \mathrm{CO}_{2}$ and for $24 \mathrm{~h}$.

The assessment of neutrophil function and survival. The degranulation of neutrophils was assayed on the basis of elastase, myeloperoxidase (MPO), and alkaline phosphatase (ALP) release and related to maximal enzyme content obtained after treatment of the cells with $0.5 \%$ Triton X-100 (Sigma-Aldrich). The assay of elastase activity was based on the cleavage of azocasein (Sigma) as a substrate at $25^{\circ} \mathrm{C}$ for $10 \mathrm{~min}$, thereafter absorbance was assessed at $490 \mathrm{~nm}$ using a BioTek EL800 (Biokom, Janki, Poland) and compared to maximal enzyme release after treatment of neutrophils with $0.5 \%$ Triton $\mathrm{X}-100$. MPO release was determined by measuring the absorbance at $490 \mathrm{~nm}$ after $10 \mathrm{~min}$ incubation of the sample with an equal volume of o-phenylendiamine (SigmaAldrich). ALP activity was estimated after $10 \mathrm{~min}$ incubation at $25^{\circ} \mathrm{C}$ with an equal volume of 4-nitrophenyl phosphate disodium salt hexahydrate (Sigma-Aldrich), then absorbance was measured at $405 \mathrm{~nm}$ and compared to maximal enzyme release after treatment of neutrophils with $0.5 \%$ Triton X-100.

Superoxide anion production was measured by a method described previously. Briefly, neutrophils were incubated with a $0.1 \%$ nitroblue tetrazolium (Sigma) solution at room temperature for $10 \mathrm{~min}$ and then the absorbance was read at $545 \mathrm{~nm} \mathrm{(12).}$ Concentrations of superoxide were calculated using the extinction coefficient $(21.1 \mathrm{nM}$

The nitric oxide (NO) level was determined by the Griess reaction described previously (12). The obtained values were expressed as a concentration of nitrite, as a stable product of NO, which accumulates in the medium. All analyses were performed in duplicate.

Neutrophil survival was determined by a 3-[4,5-dimethyl-2thiazolyl]-2,5-diphenyl-2H-tetrazolium bromide (MTT) test based on the conversion of the MTT by mitochondrial dehydrogenase to a colored formazan product as measured at absorbance of $565 \mathrm{~nm}$ (14). Cell viability, defined as a relative absorbance of each sample compared to control treated only with PBS, was calculated and expressed as a percentage of that of the control.

Statistical analysis. For all experiments with neutrophils, at least triplicate determinations were made for each experimental condition. All data are expressed as the mean \pm standard deviation (SD) for continuous variables. Comparisons between two experimental groups were performed using Student's $t$-test, for multiple group comparisons, significance was identified by one-

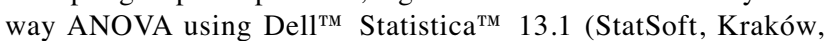
Poland). Results were considered statistically significant at $p<0.05$.

\section{Results}

Before implantation of biomaterial, elastase release from neutrophil cultures reached $50.73 \pm 2.30 \%$ of maximal release. Surgical intervention intensified this response in the sham group as well as in neutrophils isolated after implantation of alginate-based biomaterial, especially after $24 \mathrm{~h}$ incubation. The implantation of carbon fibers did not evoke pronounced elastase release at the studied time points. Elastase release by neutrophils isolated before the implantation of biomaterial, and neutrophils isolated after sham surgery, decreased under the influence of different AMP preparations at both studied time points. This effect was most pronounced after $24 \mathrm{~h}$ incubation with the F2 fraction. The suppressive effect of AMP extract on elastase release was also noted after the implantation of carbon fibers into the knee joint, but to a smaller degree. Conversely, AMP extracts did not diminish elastase release after the implantation of alginate-based biomaterial (Figure 1).

An increased MPO release after 30-min incubation was observed after sham surgery and after the implantation of both studied biomaterials. Furthermore, the neutrophil response significantly diminished after $24 \mathrm{~h}$ incubation in all study groups. This effect was enhanced after stimulation of cell cultures with AMP preparations, especially F2 (Figure 2).

Surgical intervention slightly enhanced the release of ALP after 30-min incubation. This response was more pronounced after the implantation of both studied biomaterials, and increased after $24 \mathrm{~h}$ incubation. ALP release was only slightly diminished after the treatment of cell cultures with AMP extracts. There were no significant differences in neutrophil response among all AMP fractions used for stimulation (Figure 3).

In our experiment, an insignificant increase of $\mathrm{NO}$ generation in response to both studied implants after incubation for $30 \mathrm{~min}$ and after $24 \mathrm{~h}$ was observed. In a further part of the study, a significant $(p<0.05)$ decrease of NO generation under the influence of different AMP fractions was found (Table I). The generation of superoxide changed insignificantly both in response to the implantation of biomaterials and after the addition of AMP extracts in the study groups (data not shown).

There was an insignificant decrease of neutrophil viability after the addition of the three AMP preparations to neutrophils isolated both after implantation of alginate-based biomaterial and carbon fiber biomaterial in comparison with values before implantation. As expected, the viability of neutrophils was lower after 24-h incubation in all study groups (Figure 4).

\section{Discussion}

Neutrophils are the main cells appearing in the early stages of inflammation. They are directed into the implantation site in order to neutralize the injury and prepare the tissue for selfrepair, therefore their presence is indispensable in order to start 


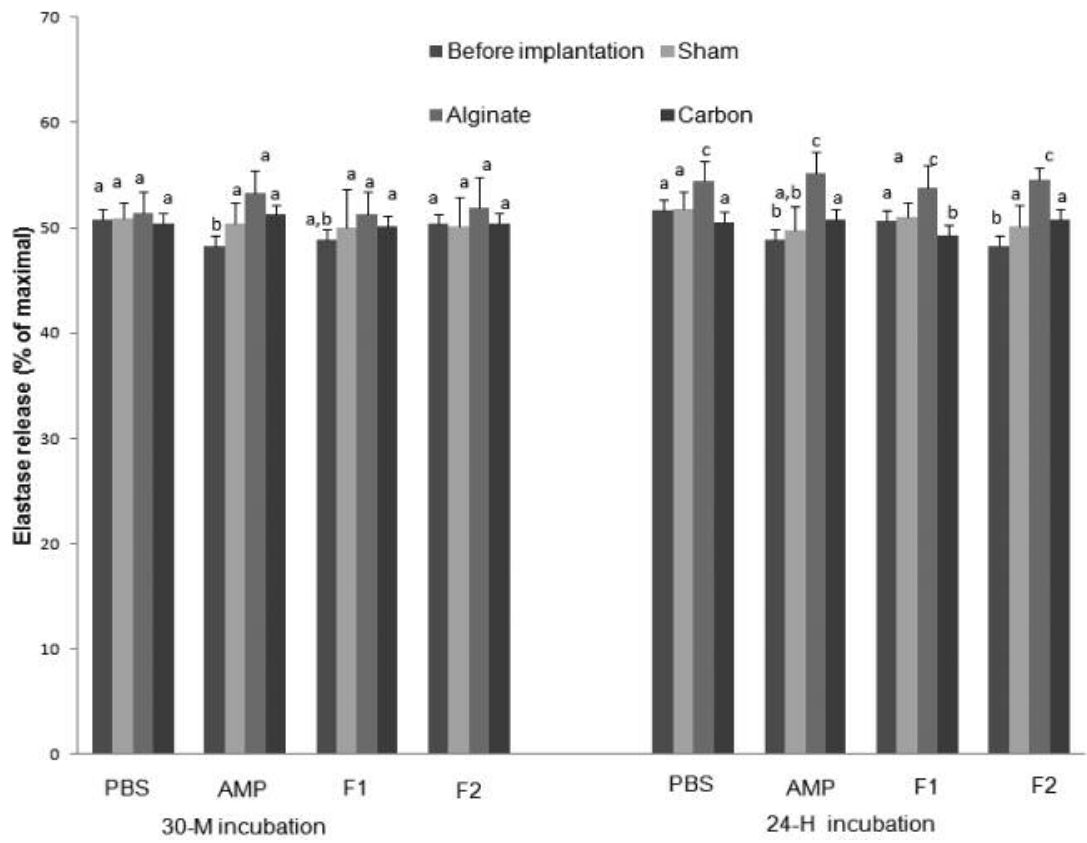

Figure 1. The effect of phosphate-buffered saline (PBS), antimicrobial peptides (AMP), fraction $1(F 1)$, and fraction $2(F 2)$ on elastase release by neutrophils after $30 \mathrm{~min}$ and $24 \mathrm{~h}$ of culture. Neutrophils were isolated before implantation (before implantation), after sham surgery (Sham), after implantation of alginate-based biomaterial into a tibial defect (Alginate) and after implantation of carbon fibers into a knee joint osteochondral defect (Carbon). Data represent the mean \pm SE of four (sham group) or five (other groups) rabbits in duplicate measurements. Mean values marked with different letters differ statistically significantly $(p<0.05)$.

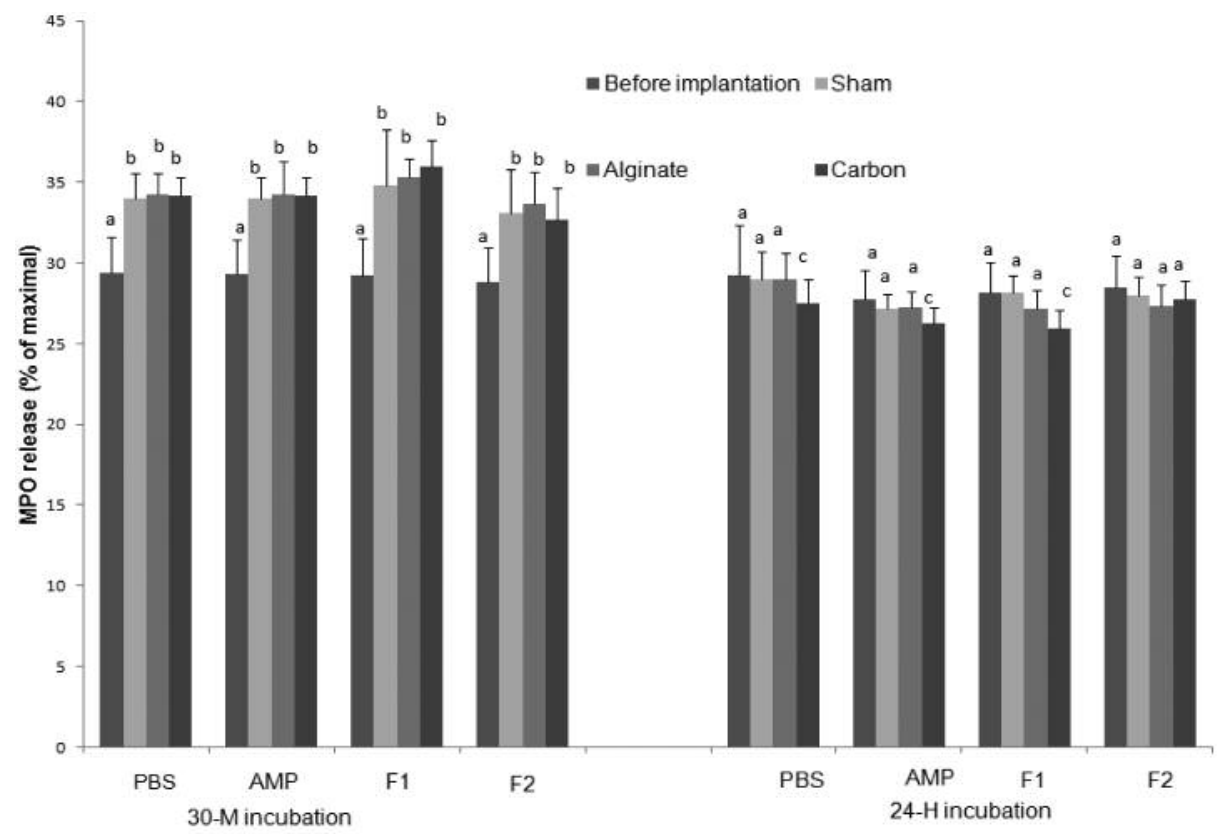

Figure 2. The effect of (PBS), antimicrobial peptides (AMP), fraction $1(F 1)$, and fraction $2(F 2)$ on myeloperoxidase (MPO) release by neutrophils after $30 \mathrm{~min}$ and $24 \mathrm{~h}$ of culture. Neutrophils were isolated before implantation (before implantation), after sham surgery (Sham), after implantation of alginate-based biomaterial into a tibial defect (Alginate) and after the implantation of carbon fibers into a knee joint osteochondral defect (Carbon). Data represent the mean \pm SE of four (sham group) or five (other groups) rabbits in duplicate measurements. Mean values marked with different letters differ statistically significantly $(p<0.05)$. 


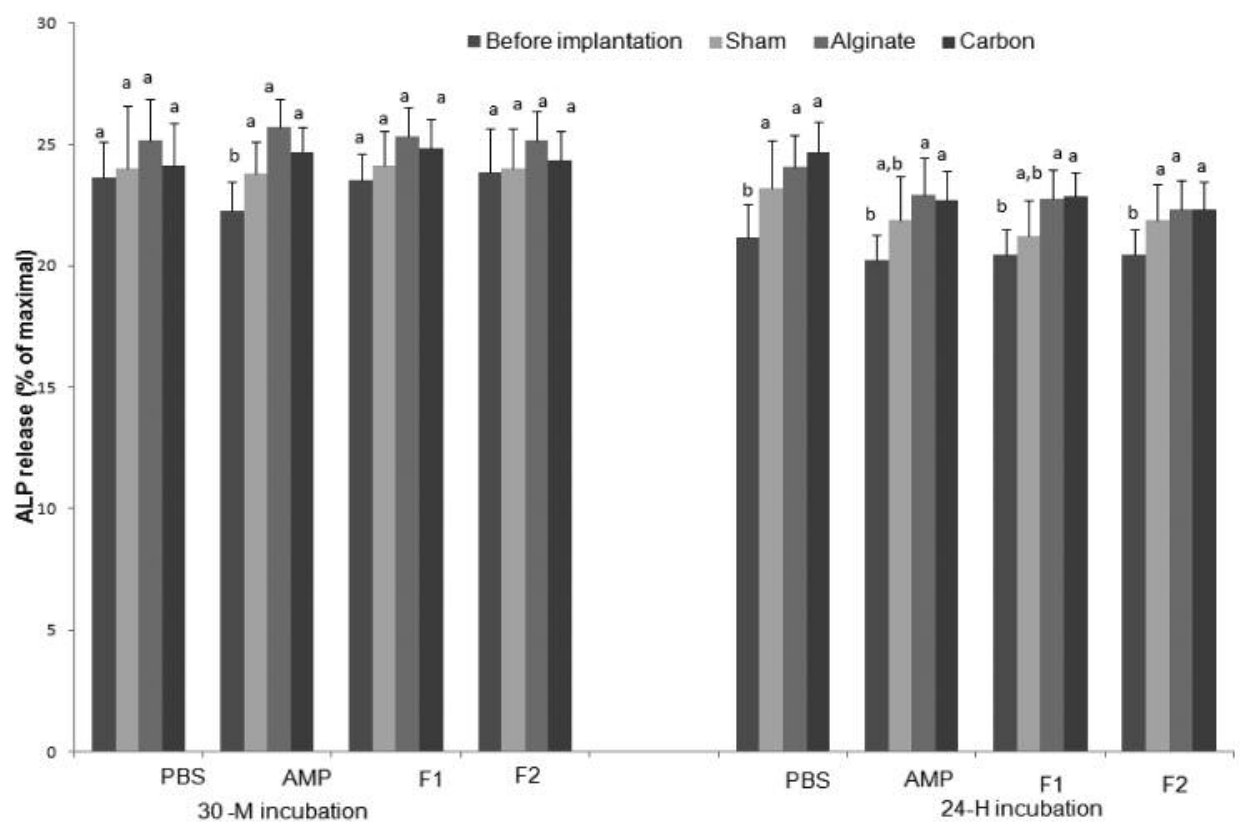

Figure 3. The effect of (PBS), antimicrobial peptides (AMP), fraction 1 (F1), and fraction $2(F 2)$ on alkaline phosphatase (ALP) release by neutrophils after $30 \mathrm{~min}$ and $24 \mathrm{~h}$ of culture. Neutrophils were isolated before implantation (before implantation), after sham surgery (Sham), after the implantation of alginate-based biomaterial into a tibial defect (Alginate) and after the implantation of carbon fibers into a knee joint osteochondral defect (Carbon). Data represent the mean \pm SE of four (sham group) or five (other groups) rabbits in duplicate measurements. Mean values marked with different letters differ statistically significantly $(p<0.05)$.

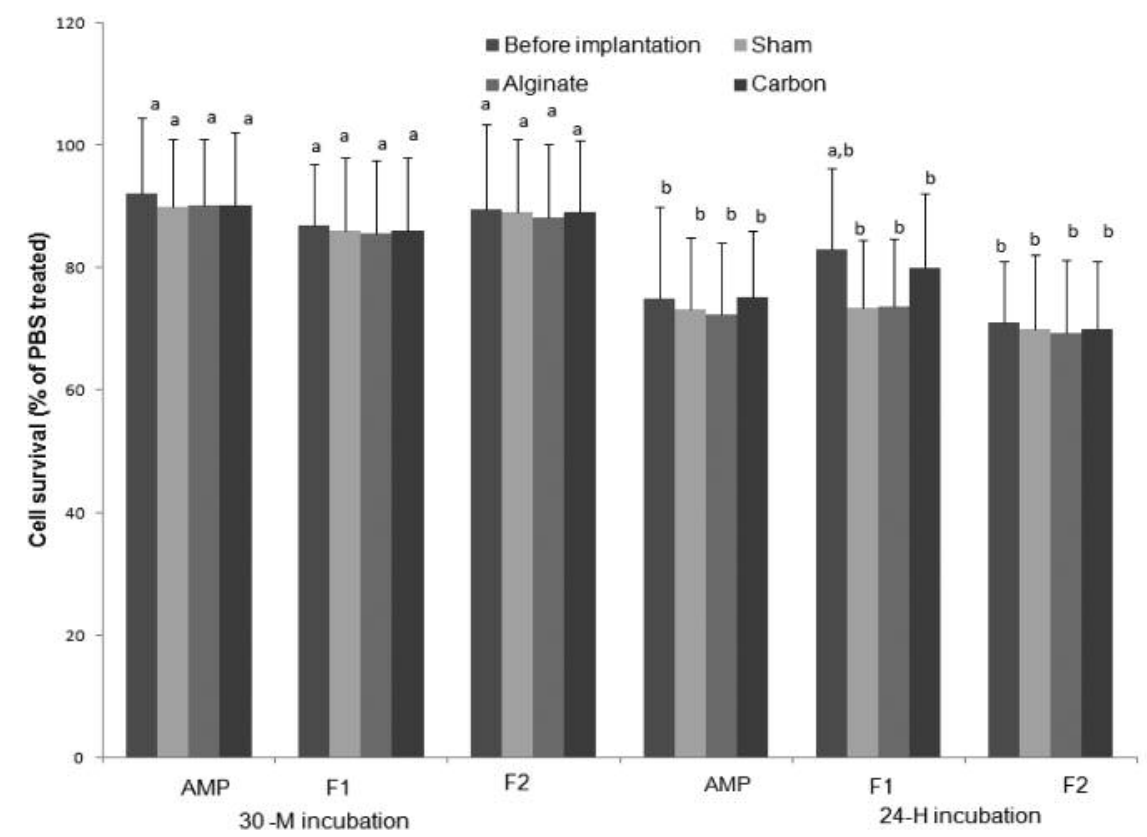

Figure 4. The effect of (PBS), antimicrobial peptides (AMP), fraction $1(F 1)$, and fraction $2(F 2)$ on cell survival on the basis of MTT reduction in neutrophils after $30 \mathrm{~min}$ and after $24 \mathrm{~h}$ of incubation expressed as a percentage of values obtained at each time point in PBS-treated cells as a control group. Data represent the mean \pm SE of four (sham group) or five (other groups) rabbits in duplicate measurements. Mean values marked with different letters differ statistically significantly $(p<0.05)$. 
Table I. The effect of phosphate-buffered saline (PBS), antimicrobial peptides (AMP), fraction 1 (F1) and fraction 2 (F2) on nitric oxide generation $\left(\mu M / 10^{6}\right.$ cells) by neutrophils after $30 \mathrm{~min}$ and $24 \mathrm{~h}$ of culture. Neutrophils were isolated before implantation (before implantation), after sham surgery (Sham), after the implantation of alginate-based biomaterial into a tibial defect (Alginate) and after the implantation of carbon fibers into a knee joint osteochondral defect (Carbon). Data represent the mean \pm SE of groups in duplicate measurements. *Statistically significantly different from the PBS-treated group $(p<0.05)$.

\begin{tabular}{|c|c|c|c|c|c|c|c|c|}
\hline \multirow[b]{3}{*}{ Treatment } & \multicolumn{4}{|c|}{ 30-Min incubation } & \multicolumn{4}{|c|}{ 24-H incubation } \\
\hline & Before implantation & & After implantatio & & Before implantation & & After implantatior & \\
\hline & $(n=14)$ & Sham $(n=4)$ & Alginate $(n=5)$ & Carbon $(n=5)$ & $(n=14)$ & Sham $(n=4)$ & Alginate $(n=5)$ & Carbon $(n=5)$ \\
\hline PBS & $3.61 \pm 0.98$ & $3.6 \pm 1.0$ & $3.7 \pm 0.64$ & $3.5 \pm 0.50$ & $3.09 \pm 1.12$ & $4.0 \pm 0.45$ & $4.17 \pm 0.6$ & $4.00 \pm 0.2$ \\
\hline AMP & $3.58 \pm 0.39$ & $3.62 \pm 0.2$ & $3.53 \pm 0.84$ & $3.67 \pm 0.18$ & $2.53 \pm 1.46^{*}$ & $2.18 \pm 1.2$ & $3.3 \pm 0.61 *$ & $3.27 \pm 0.15^{*}$ \\
\hline F1 & $3.55 \pm 0.48$ & $3.5 \pm 0.5$ & $3.72 \pm 0.94$ & $3.6 \pm 0.87$ & $2.55 \pm 1.02 *$ & $2.6 \pm 1.5$ & $3.61 \pm 0.48^{*}$ & $3.87 \pm 0.3 *$ \\
\hline F2 & $3.43 \pm 0.45$ & $3.5 \pm 0.22$ & $3.3 \pm 0.69$ & $3.2 \pm 0.7$ & $2.22 \pm 0.98 *$ & $2.17 \pm 1.8$ & $3.38 \pm 0.72 *$ & $3.12 \pm 0.21 *$ \\
\hline
\end{tabular}

the healing process (15). Our study revealed that the implantation of both alginate-based biomaterial and carbon fibers evoked an increased neutrophil secretory response. This response was partially restricted by treatment of the neutrophil cultures with different fractions of AMP. Aarbiou et al. showed both human defensin HNP1-3 and human cathelicidin LL-37 induced cytochrome $c$ release from mitochondria within $3 \mathrm{~h}$ after stimulation (9). These components induced cell death, which is associated with mitochondrial injury and mediated via different intracellular pathways. We observed a significant decrease in neutrophil viability after 24-h incubation, additionally, the viability was slightly lower in cultures stimulated with AMP extracts. In a previous study, Nagaoka et al. reported some AMP extract components to be proapoptotic factors (16). According to Semple et al., human defensins have an immunosuppressive effect, especially on macrophages (17). The specific effect of components of rabbit AMP extract has not been evaluated to date.

The increased neutrophil activity observed in our study after the sham surgery was a response to surgical trauma. Surgical trauma during implantation is a short-lived but strong stimulus of inflammation. The presence of a foreign body in a surgical wound can activate immune processes, stimulate inflammatory mechanisms and prolong their duration. The course of these complex processes and the fate of the implant depend on the balance between the severity of the inflammatory response and the extent of repair processes. According to Lenz et al., after trauma, neutrophils were primed for the release of pro-inflammatory cytokines and elastase (4). We noted an increase in neutrophil secretory response after trauma associated with drilled lesions (the sham group), as well as in the experimental groups (both alginate-based and carbon fiber biomaterials).

In a study by Lins et al., it was found that alginates have a pro-inflammatory effect mainly on macrophages, but also on neutrophil activation. According to these authors, alginates induce monocyte and macrophage stimulation through nuclear factor kappa light-chain enhancer of activated B-cells involved pathway, resulting in an increase of cytokine production. Macrophage stimulation seems to be related to the ability of alginate to enhance the healing process (18). However, the neutrophil response to these materials is still poorly understood. Our experiment confirmed increased degranulation of neutrophils after implantation of alginate-based biomaterial. Neutrophils produce ROS, degranulate to release proteins into the extracellular milieu, and secrete a variety of cytokines following activation (5). This effect has also been confirmed as the result of the interaction of cultured human neutrophils with biomaterials occurring in the form of ROS production, the release of granule proteins (MPO and matrix metalloprotease-9), and the secretion of some cytokines as well as neutrophil peptides (5). We found that implantation of alginate-based biomaterial evoked increased release of neutrophil enzymes. Similarly, NO generation in response to this biomaterial was also increased, especially in the second measurement. In light of the fact that alginates are susceptible to degradation by ROS in a process known as 'free radical depolymerization' or 'oxidative-reductive depolymerization' (19), the evaluation of substances which attenuate the excessive neutrophil release would be of great interest.

In some aspects of tissue engineering, it may be beneficial to induce local inflammation in order to stimulate angiogenesis, whereas in other areas, local inflammation may lead to the formation of fibrous capsules that reduce the diffusion of nutrients and oxygen to cells within the biomaterial (19). However, both cases, it is important to avoid chronic inflammation (19). For this purpose, we evaluated different fractions of AMP extract as the means for potential diminution of inflammatory response during the implantation of biomaterial in order to prevent the 
deleterious effect involved in the interactions of immune cells with biomaterial.

On the basis of cultured neutrophil response, we found that carbon fibers caused an inflammatory reaction, with increased MPO release and NO generation, which was ameliorated after contact with AMP extracts. To our knowledge, this is the first study on this topic. Previously only histological studies were carried out. According to Rajzer et al., 7 days after implantation into rat skeletal muscles, neutrophils and eosinophils were observed around implanted carbon fibers. The presence of cells responsible for combating infections provides information on the inflammatory infiltration associated with both the presence of a foreign body and tissue injury as an effect of the implantation (15).

Our study described for the first time the use of two kinds of biomaterial implanted into two different tissues for comprehensive evaluation of neutrophil response during the first inflammatory reaction to an implant. The standard method of estimation of biomaterial biocompatibility is the assessment of implant-tissue interactions based on microscopic evaluation, amongst others. During this evaluation, histological preparations are examined in regard to tissue response on the basis of the presence of granulocytes, lymphocytes and macrophages in histological sections. Our study permits the assessment of neutrophil response to an implant without the necessity for preparation of histological sections from material obtained post mortem. Additionally, we determined that autologous neutrophil extract consisting of AMP diminished the pro-inflammatory response and may, after further in vivo studies, be considered as an inhibitor of foreign body response caused by neutrophils in the early inflammatory phase.

\section{References}

1 Selders SG, Fetz AE, Radic MZ and Bowlin GL: An overview of the role of neutrophils in innate immunity, inflammation and host-biomaterial integration. Regen Biomater 4(1): 55-68, 2017.

2 Jhunjhunwala S, Aresta-DaSilva S, Tang K, Alvarez D, Webber MJ, Tang BC, Lavin DM, Veiseh O, Doloff JC, Bose S, Vegas A, Ma M, Sahay G, Chiu A, Bader A, Langan E, Siebert S Li J, Greiner DL, Newburger PE, von Andrian U H, Langer R and Anderson DG: Neutrophil responses to sterile implant materials. PLoS One 10(9): e0137550, 2015.

3 Schmidt-Bleek K and Schell H, Schulz N, Hoff P, Perka C, Buttgereit F, Volk H-D, Lienau J and Duda GN: Inflammatory phase of bone healing initiates the regenerative healing cascade. Cell Tissue Res 347: 567-573, 2012.

4 Lenz A, Franklin GA and Cheadle WG: Systemic inflammation after trauma. Injury Int J Care Injured 38: 1326-1345, 2007.

5 Jhunjhunwala $S$ : Neutrophils at the biological-material interface. ACS Biomater Sci Eng 4: 1128-1136, 2018.
6 McPhee JB and Hancock REW: Function and therapeutic potential of host defence peptides J Pept Sci 11: 677-687, 2005.

7 Alalwani SM, Sierigk J, Herr C, Pinkenburg O, Gallo R, Vogelmeier C and Bals R: The antimicrobial peptide LL-37 modulates the inflammatory and host defense response of human neutrophils. Eur J Immunol 40: 1118-1126, 2010.

8 Barlow PG, Li Y, Wilkinson TS, Bowdish DME, Lau E, Cosseau C, Haslett C, Simpson AJ, Hancock REW and Davidson DJ: The human cationic host defense peptide LL-37 mediates contrasting effects on apoptotic pathways in different primary cells of the innate immune system. J Leukoc Biol 80: 509-520, 2006.

9 Bowdish DME, Davidson DJ and Hancock REW: Immunomodulatory properties of defensins and cathelicidins. In: Antimicrobial Peptides and Human Disease. Shaffer W (ed.), pp. 27-66, 2006.

10 Aarbiou J, Tjabringa GS, Verhoosel RM, Ninaber DK, White SR, Peltenburg LTC, Rabe KF and Hiemstra PS: Mechanisms of cell death induced by the neutrophil antimicrobial peptides $\alpha$ defensins and LL-37. Inflamm Res 53: 119-127, 2006.

11 Szponder T, Wessely-Szponder J and Smolira A: Evaluation of platelet-rich plasma and neutrophil antimicrobial extract as two autologous blood-derived agents. Tissue Eng Regen Med 14(3): 287-296, 2017.

12 Wessely-Szponder J, Szponder T and Bobowiec R: Different activation of monocyte-derived macrophages by antimicrobial peptides at a titanium tibial implantation in rabbits. Res Vet Sci 115: 201-210, 2017.

13 Stodolak E, Paluszkiewicz C, Bogun M and Błażewicz M: Nanocomposite fibres for medical applications. J Mol Structure 924-926: 208-213, 2009.

14 Kakuta Y, Aoshiba K and Nagai A: C-Reactive protein products generated by neutrophil elastase promote neutrophil apoptosis. Arch Med Res 37: 456-460, 2006.

15 Rajzer I, Menaszek E, Bacakova L R, and Blazewicz M: In vitro and in vivo studies on biocompatibility of carbon fibres. J Mater Sci: Mater Med 21: 2611-2622, 2010.

16 Nagaoka I, Kaori S, Niyonsaba F, Tamura H and Hirata M: Modulation of neutrophil apoptosis by antimicrobial peptides. ISRN Microbiology 2012: 345791, 2012.

17 Semple F, Webb S, Li H-N, Patel HB, Perretti M, Jackson IJ, Gray M, Davidson DJ, and Dorin JR: Human b-defensin 3 has immunosuppressive activity in vitro and in vivo. Eur J Immunol 40: 1073-1078, 2010.

18 Lins KOAL, Vale MI, Ribeiro RA and Costa-Lotufo LV: Proinflammatory activity of an alginate isolated from Sargassum vulgare. Carbohydr Polymers 92: 414-420, 2013.

19 Andersen T, Strand BL, Formo K, Alsberg E and Christensen BE: Alginates as biomaterials in tissue engineering. Carbohydr Chem 37: 227-258, 2012. 